\title{
Deuterium retention in Rhenium-Doped Tungsten
}

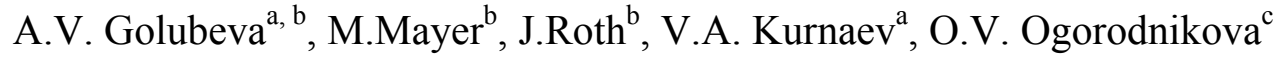 \\ ${ }^{a}$ Moscow Physics’ and Engineering Institute, , Kashirsky str. 31, Moscow, Russia, 115409 \\ ${ }^{b}$ Max-Planck-Institut für Plasmaphysik, EURATOM Association, Boltzmannstr. 2, D-85748 \\ Garching, Germany \\ ${ }^{c}$ CEA Cadarache, Bat. 506, F-13108 Saint Paul Lez Durance cedex, France
}

\begin{abstract}
Deuterium retention in rhenium doped tungsten (W-Re) was investigated by means of the thermodesorption technique. W-Re alloys of different structures and concentrations of Re were investigated. All tungsten rhenium alloys had a common structural feature micron size voids in the bulk. The samples were irradiated by $200 \mathrm{eV}$ deuterium ions in the fluence range $10^{22} \div 10^{24} \mathrm{D} / \mathrm{m}^{2}$. The thermodesorption spectra of polycrystalline $\mathrm{W}$ measured with a linear ramp of $5 \mathrm{~K} / \mathrm{s}$ has two peaks at $\sim 450$ and $650 \mathrm{~K}$. In TDS of W-Re alloys an additional high-temperature peak was found at $850 \mathrm{~K}$. The observed retention in W-Re alloys depends strongly on materials structure. In the range of 1-10\% Re admixture the Re concentration in W-Re alloy doesn't influence noticeably the deuterium retention. Structure changes (filling of voids in material) was found to take place both at annealing and low-energy deuterium irradiation.
\end{abstract}

PACS:

61.72.Qq, 61.72.-y, 61.82.Bg, 61.80.Jh, 81.40.Wx

JNM keywords: Tungsten, Tungsten Alloys and Compounds, Voids, Deuterium PSI-17 keywords: Deuterium inventory, Retention, Tungsten 
*Corresponding author address: 115409 Kashirskoe shosse, 31, Moscow, Russia

*Corresponding author E-mail: anna-golubeva@yandex.ru

Presenting author: Anna Golubeva

Presenting author e-mail: anna-golubeva@yandex.ru

\section{Introduction}

Hydrogen interaction with plasma-facing materials (PFM) of fusion devices is of interest and importance from both fuel balance and radiation safety points of view. Being one of the candidate PFMs, tungsten is an object of extensive studies of hydrogen material interaction. Although a large database on the subject was collected, there are still many open questions in different aspects of $\mathrm{H}-\mathrm{W}$ interaction. One of them is the influence of rhenium impurity on hydrogen retention in the material. Rhenium can be added into tungsten intentionally to improve mechanical properties of the material. Also, irradiation of PFM with neutrons produced in thermonuclear reactions will cause the transmutation of the whole elements of PFM. For tungsten, the transmutation into rhenium will occur. For a typical neutron spectrum of fusion device the following path of transmutation are the most probable:

$$
\text { 1) } \begin{aligned}
{ }^{184} \mathrm{~W}(\mathrm{n}, \gamma){ }^{185} \mathrm{~W} & \rightarrow(\beta-)^{185} \operatorname{Re}(\mathrm{n}, \gamma){ }^{186} \mathrm{Re} \\
\text { 2) } \quad{ }^{186} \mathrm{~W}(\mathrm{n}, \gamma){ }^{187} \mathrm{~W} & \rightarrow(\beta-)^{187} \operatorname{Re} \rightarrow(\mathrm{n}, \gamma){ }^{188 \mathrm{~m}} \mathrm{Re} \rightarrow(\gamma){ }^{188} \mathrm{Re} \\
& \rightarrow(\mathrm{n}, 2 \mathrm{n}){ }^{186} \mathrm{Re}
\end{aligned}
$$

According to estimations for an industrial fusion reactor [1], after 2.5 years of operation the plasma-facing tungsten will contain up to $4 \% \mathrm{Re}$, and after 10 years of operation rhenium concentration can increase up to 8 percent. For the ITER reactor, the total duration of thermonuclear burn will be much less than in the case of an industrial reactor, and one may expect $\sim 1 \%$ of rhenium in the initial tungsten armour. 
Change of the material composition can influence hydrogen trapping in tungsten. Until now, there are no studies available on the subject. The present work was performed to obtain information about the influence of rhenium admixture on hydrogen accumulation in tungsten.

\section{Experimental}

\subsection{Materials under investigation}

Deuterium retention was investigated in the following tungsten materials:

- polycrystalline tungsten (PolyW). The samples were cut from $99.96 \%$ purity tungsten foils produced by Plansee-AG. After pre-annealing at $1500 \mathrm{~K}$ for three hours PolyW consists of grains with sizes 5-15 $\mu \mathrm{m}$. The material had no void.

- W-Re alloys with 1, 5 and 10\% concentration of Re, produced by Plansee AG. All three materials, independently on Re contamination have the same structure. The grain sizes of these materials as received are in the range $10-25 \mu \mathrm{m}$. A common feature for all three Plansee W-Re alloys is the presence of voids in the bulk. The typical size of void is $1-2 \mu \mathrm{m}$. The amount of voids was estimated to be about $6 \%$ of the volume.

- $\quad \mathrm{W}-25 \%$ Re alloy produced by Institute of Rare Earth Metals, Russia, by powder metallurgy and hot rolling. This material has a void percentage of about $1 \%$ of volume. After pre-annealing at $1500 \mathrm{~K}$ during 3 hours the crystallites size is about 20-50 $\mu \mathrm{m}$, the voids size $-1-10 \mu \mathrm{m}$.

According to [2], the phase of $\mathrm{Re}$ in $\mathrm{W}$ is solution if Re content in W-Re alloy is less than $30 \%$. The material is "tungsten-like", with the same structure as pure tungsten (bcc). The atomic radiuses of the elements are close, so the lattice parameter do not vary significantly with composition. For W-25\%Re alloy several percent of $\sigma$-phase incorporation is possible. 
All samples had dimensions of $10 \times 15 \mathrm{~mm}$ and thicknesses in the range $0.5-1 \mathrm{~mm}$. After cutting all samples were mechanically polished and electro polished. PolyW and W$25 \% \mathrm{Re}$ were also pre-annealed in vacuum at $10^{-5} \mathrm{mbar}$ at $1500 \mathrm{~K}$ during three hours. This procedure is widely used for reducing the number of intrinsic defects present in tungsten after manufacturing $[3,4]$ and usually led to growth of the grain sizes. Most of Plansee WRe samples were used as received. Some of them were also pre-annealed, that led to unexpected result of structure change, described below.

\subsection{Experimental Set-Up}

The experimental investigations of deuterium retention in tungsten materials were carried out at the „High current ion source“ [5]. After placing in the interaction chamber, every sample was annealed at $1200 \mathrm{~K}$ during 10 minutes to remove surface impurities.

The tungsten samples were irradiated by $\mathrm{D}_{3}{ }^{+}$with $\mathrm{E}_{0}=600 \mathrm{eV}$, corresponding to 200 $\mathrm{eV}$ per deuteron. In order to obtain higher ion fluxes in the case of low-energy irradiation, $3.6 \mathrm{keV} \mathrm{D}_{3}{ }^{+}$ions were extracted from the ion source and decelerated to $600 \mathrm{eV}$ by a positive sample biasing. A fraction of the incident ions can be neutralized at the entrance apertures, resulting in $1.5-3 \%$ fraction of high-energy neutrals in the decelerated beam [6, 7]. This leads to a $10 \%$ uncertainty of the low-energy particles fluence [8]. Ion implantations were always performed at normal incidence and room temperature. The flux at the sample surface was $\sim 5 \cdot 10^{19} \mathrm{D} / \mathrm{m}^{2} \mathrm{~s}$.

Deuterium retention in tungsten materials was investigated by means of the thermodesorption technique. After implantation the sample was heated by electron impact with a linear heating ramp of $4.5 \mathrm{~K} / \mathrm{s}$. The sample temperature was measured by an infrared pyrometer. The signals of $\mathrm{D}_{2}, \mathrm{HD}$ and several hydrocarbon gases were registered by a quadrupole mass-analyzer, calibrated as described in [9] by desorption of implanted deuterium from titanium. Depending on vacuum condition, the fraction of deuterium 
released as HD molecules is between 5 and $30 \%$. The fraction of deuterium atoms released as hydrocarbons is negligible.

\section{Results and discussion}

\subsection{Thermodesorption spectra}

Thermodesorption spectra (TDS) of the three Plansee W-Re alloys show no pronounced difference for various rhenium concentration. Thermodesorption spectra of W$5 \%$ Re and PolyW implanted with the same fluence of $10^{24} \mathrm{D} / \mathrm{m}^{2}$, normalized to the height of the first peak are presented in Fig. 1.a. The TDS of PolyW shows a two-peaks structure with peaks at $400 \mathrm{~K}$ and $650 \mathrm{~K}$. TDS of W-Re alloy contains an additional peak at $850 \mathrm{~K}$.

Until now there is no agreement among investigators in the understanding of the nature of the TDS peaks of tungsten, number of peaks and their positions, as hydrogen retention in tungsten is strongly influenced by the material structure and history. Hydrogen retention can differ in several times for two tungsten materials of the same $99.96 \%$ purity but different structure [10].

The existence of several types of traps for hydrogen in tungsten is reported. Most often two type of traps are mentioned, with binding energies of 0.5-0.9 and 1.2-1.5 eV. For a linear ramp of $\sim 5 \mathrm{~K} / \mathrm{s}$ as used in the present experiments the two types of traps result in peaks at temperatures about 400 and $650 \mathrm{~K}$ [9]. There is no common opinion about the nature of this traps. The first peak at $\sim 400 \mathrm{~K}$ can be associated with some defects of the tungsten lattice (grain boundaries, dislocations, impurities, presence of bulk oxide $[11,9]$ ). Other authors suppose that this peak corresponds to diffusion limited release of deuterium from interstitial sites in the lattice [12]. The second peak is usually supposed to correspond to initial vacancies and lattice defects created during ion bombardment (vacancies, bubbles, vacancies clusters [13]), as the peak grows after creating defects in tungsten by energetic ions pre-implantation [9]. Some authors also reported the existence of higher energy traps. 
Van Veen et al. [14] registered a high-temperature peak at $800 \mathrm{~K}$ in the TDS of monocrystalline tungsten irradiated by $200 \mathrm{eV}$ deuterons. Before deuterium implantation the sample was pre-implanted by $6 \mathrm{MeV}$ protons and $\varnothing 1 \mathrm{~nm}$ voids were created in the material. This peak at $800 \mathrm{~K}$ was associated with deuterium release from small gas bubbles. In [15] the hydrogen binding energy in chemisorbed state on bubbles walls in monocrystalline $\mathrm{W}$ was found to be $2.1 \mathrm{eV}$, corresponding to an annealing temperature of 900K. An additional peak appears at $1000 \mathrm{~K}$ in the TDS of plasma-sprayed tungsten using the same heating ramp as in present work. Plasma-sprayed tungsten contains $8 \%$ voids in the bulk. This peak is explained by the presence of voids in the material [10].

The position of the third high-temperature peak in TDS and it's amplitude does not differ significantly for the three Plansee W-Re alloys, which have different rhenium content, but the same structure. Therefore, one may suppose the presence of the third peak is a result of capture in some defects of crystalline structure, but not of binding with Re impurity.

For a quantitative comparison, thermodesorption spectra (TDS) of PolyW and W$1 \%$ Re Plansee alloy irradiated with a fluence of $10^{22} \mathrm{D} / \mathrm{m}^{2}$ with $200 \mathrm{eV}$ deuterium are shown in Fig. 1.b. The amount of deuterium retained in W-Re is much higher than in polycrystalline tungsten. The amplitudes of the first two peaks in TDS of W-Re are higher, which means that Plansee W-Re alloys contain large amount of hydrogen trapping sites.

\subsection{Retention}

The fluence dependencies of deuterium retention in all tungsten materials under investigation are presented in Fig. 2. In the fluence interval $10^{22}-10^{24} \mathrm{~m}^{2}$ no saturation of deuterium accumulation was observed. The difference in total retention for Plansee W-Re materials with 1,5 and $10 \%$ is not significant. This means that for $1-10 \%$ of Re admixture 
the Re contamination does not influence pronouncedly the deuterium accumulation in the material.

The retention in Plansee W-Re alloys is higher than in PolyW. The difference at low fluences is more than one order of magnitude, becoming smaller with increasing fluence. The retention in $\mathrm{W}-25 \% \mathrm{Re}$ alloy produced by Rare Earth Metals Institute, on the contrary, is very close to the retention in PolyW.

It shows clearly, that not the presence of Re is responsible for the higher retention in the Plansee W-Re alloys, but the concentrations of defects of different types. Therefore, the production of rhenium in tungsten due to transmutation under neutron irradiation will not influence noticeably hydrogen isotopes accumulation in the tungsten armour of a fusion device.

\subsection{Structural change of W-Re alloys under annealing and low-energy ion bombardment}

An interesting effect of material structural change was found after annealing W-Re Plansee alloys in vacuum at $1500 \mathrm{~K}$. On the Fig. 3. the scanning electron microscope (SEM) images of surfaces of $\mathrm{W}-1 \%$ Re samples before and after annealing at $1500 \mathrm{~K}$ during 3 hours are shown. Unusual for $\mathrm{W}$, no growth of the grains was observed. Instead, the annealing causes diffusion of $\mathrm{W}$ and Re atoms to fill the voids in the bulk of W-Re alloys. EPMA analysis shows, that rhenium contamination in material, which fills the void, is the same as in the whole sample, so no preferentially diffusion of rhenium or tungsten was observed. For the annealed sample, the micron size voids are completely filled with material. Cross-section microscopy shows that this change of structure occurs through the whole bulk of the $\mathrm{W}-1 \%$ Re sample.

It is interesting, that the effect of irradiation of W-1\%Re sample with $10^{24} \mathrm{D} / \mathrm{m}^{2}$ of $200 \mathrm{eV}$ deuterium is absolutely the same. The lower the fluence of $200 \mathrm{eV}$ deuteron is 
achieved, the less pronounced is the effect of voids filling. The voids filling process it was found to become slower with increasing rhenium concentration.

After W-5\%Re irradiation with the same fluence of $200 \mathrm{eV}$ deuterons the voids are filled with material only in the area of a beam spot. This confirms that the void filling is a result of low energy deuterium irradiation. Cross-section microscopy shows that voids are filled with material up to depths of hundreds $\mu \mathrm{m}$. This is not only much larger than the implantation range, but also than the diffusion range of deuterium. The reason for the effect of voids filling is not clear. It can be stresses in the lattice in the near-surface region due to the large deuterium concentration during implantation, which creates distortions in lattice structure far beyond the implantation zone and causes diffusion of matrix atoms..

The void filling with material (both after annealing and low-energy deuterium irradiation) leads to significant decrease of the $850-\mathrm{K}$ peak in TDS of W-Re alloys confirming that the voids are responsible for this peak. Due to the manufacturing process of W-Re alloys (powder metallurgy followed by hot-rolling) the presence of an oxide layer on the surfaces of voids is possible. The state in which deuterium is trapped could be adsorption of deuterium on the walls of voids.

The total amount of deuterium retained in $\mathrm{W}-1 \% \mathrm{Re}$ is decreased by the annealing procedure not more than on $40 \%$ pointing that the amount of such defects like grain boundaries and dislocations is not strongly deceased and that mainly the energy introduced in the material was spent on voids filling.

\section{Conclusions}

Deuterium retention in tungsten-rhenium alloys as compared with polycrystalline tungsten at room temperature was investigated experimentally by means of thermodesorption spectroscopy. 
With a heating ramp of $4.5 \mathrm{~K} / \mathrm{s}$ TDS of W-Re alloys a high-temperature peak was found at $\mathrm{T} \approx 850 \mathrm{~K}$ in TDS spectra of W-Re alloys. This peak is absent in TDS of polycrystalline $\mathrm{W}$. The corresponding trapping site is supposed to be chemisorptions on the walls of voids in the bulk.

An effect of voids filling in W-Re alloys at low-energy deuterium irradiation was found, the most pronounced at low Re content.

Deuterium retention in W-Re alloys is strongly influenced by the material structure. For Re concentrations from 1 to $10 \%$ no pronounced influence of rhenium contamination on deuterium retention was found. It can be assumed that an admixture of Re in tungsten armour will not influence significantly the accumulation of hydrogen isotopes in tungsten. Damage of material due to neutrons irradiation accompanying the transmutation, or imperfections of crystalline structure of Re-doped tungsten in case of intentional adding of Re in material may significantly increase hydrogen isotopes trapping.

\section{Acknowledgments}

One of the authors (A. V. Golubeva) is very grateful to the Max-Planck society for the possibility of performing the present work. The authors want to express their gratitude to A. Weghorn for his technical assistance and to S. Lindig, G. Matern, and J. Perchermaier for their kind help with sample preparation and SEM analysis. 

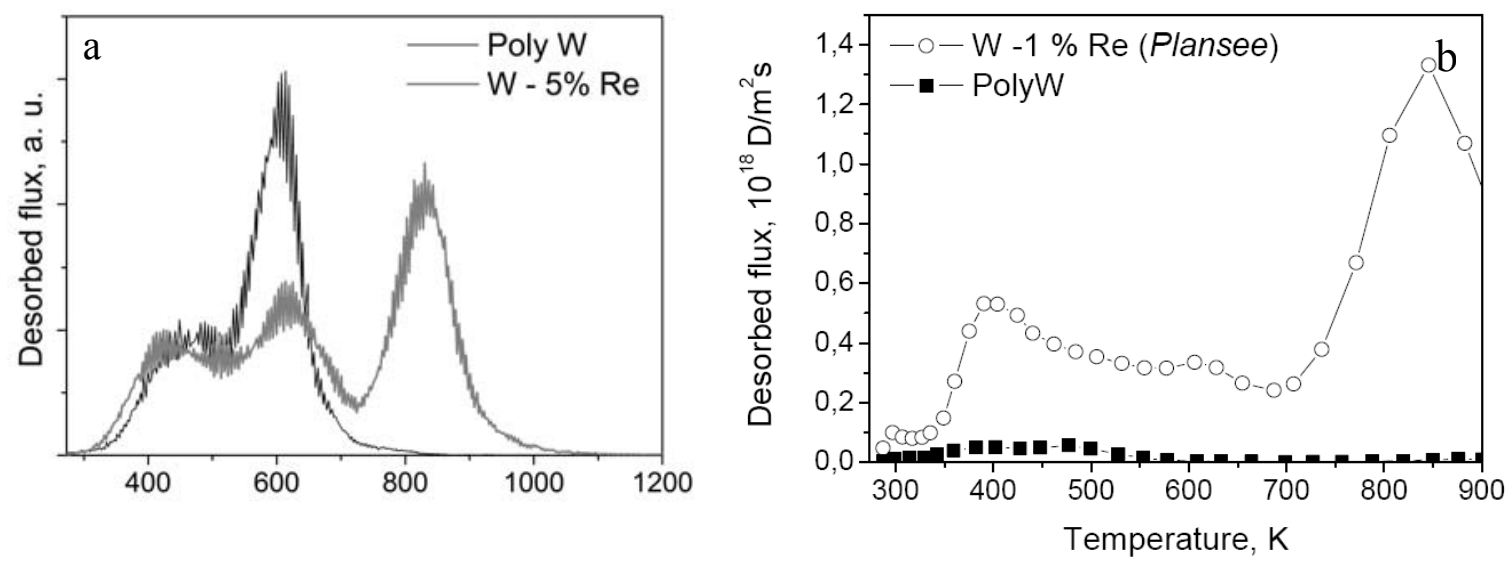

Fig. 1. TDS of PolyW and W-Re (Plansee). a) - TDS of PolyW and W-1\%Re, implanted with a fluence of $10^{22} \mathrm{D} / \mathrm{m}^{2}$ of $200 \mathrm{eV}$ deuterium. The rate of the TDS ramp was 4.5 K/s b) - TDS of PolyW and W-5\%Re, implanted with $10^{24} \mathrm{D} / \mathrm{m}^{2}$, normalized to the first TDS peak. The rate of the TDS ramp was $0.1 \mathrm{~K} / \mathrm{s}$ 


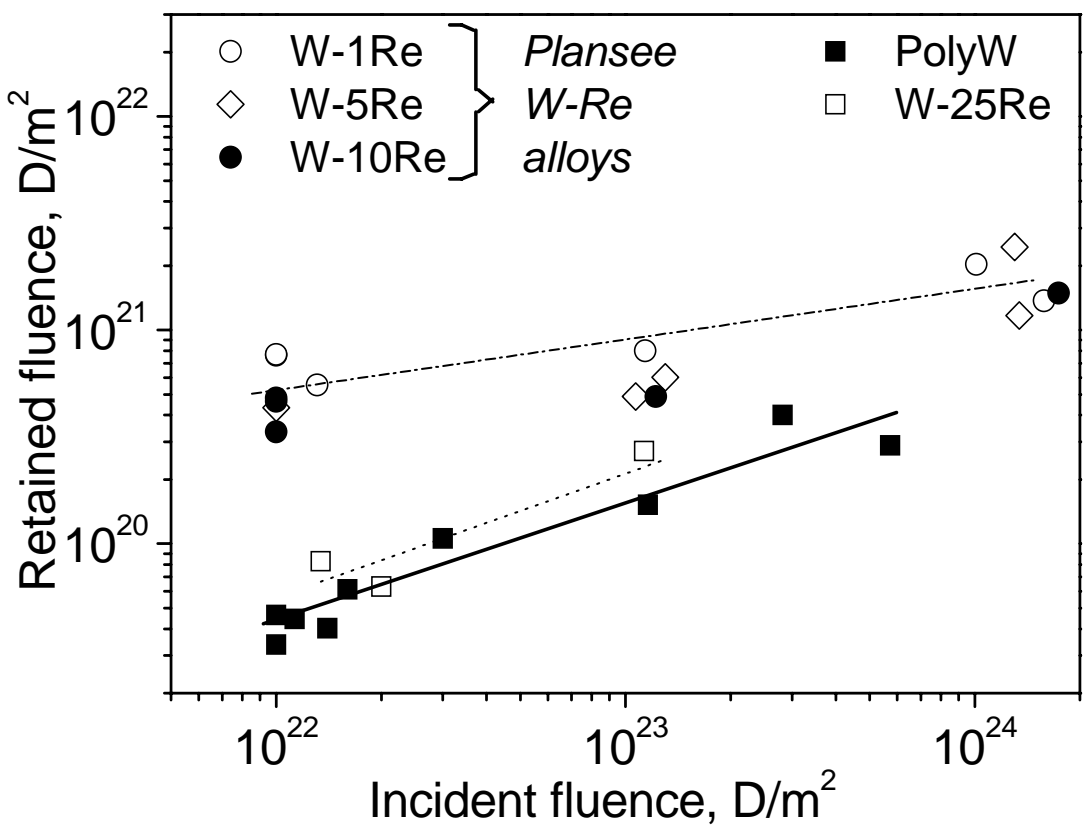

Fig. 2. Fluence dependence of $200 \mathrm{eV}$ deuterium retention in PolyW, W-Re alloys produced by Plansee AG, Austria, and W-25\%Re from Rare Earth Metals Institute, Russia 


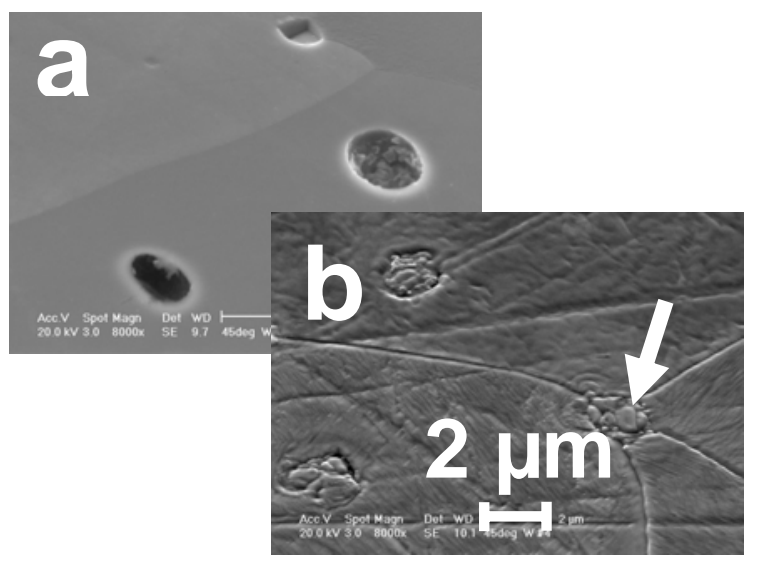

Fig. 3. SEM images of Plansee W-1\%Re surface a) before annealing b) after 3 hours annealing at $1500 \mathrm{~K}$. Images were obtained with the same magnification of SEMs 


\section{References}

1. R. Pampin, «Tungsten transmutation and resonance self-shielding in PPCS models for the study of sigma-phase formation», UKAEA FUS 525, EURATOM/UKAEA Fusion, 2005

2. Phase Diagrams of Binary Tungsten Alloys, edited by S.V Navenger Naidu and P. Rama Rao, 1991

3. R. A. Anderl, D. F. Hooland, G. R. Longhurst, R. J. Pawelko, C. L. Trybus, C. H. Sellers, Fusion Technology, Vol.21 (1992) 745

4. O. V. Ogorodnikova, J. Roth and M. Mayer, J. Nucl. Mater., 313-316 (2003) 469

5. Eckstein, W., Garcia-Rosales, C., Roth, J., and Ottenberger, W., "Sputtering data", IPPreport 9/82, 1993.

6. Bay, H.L., Roth, J., and Bohdansky, J., J. of Applied Physics, Vol. 48, No 11 (1977) 4722.

7. M. Balden, and J. Roth, J. Nucl. Mater., 280 (2000) 39.

8. Staudenmaier, G., Roth, J., Behrisch, R., Bohdansky, J., Eckstein, W., Staib, P., J. Nucl. Mater., 84 (1979) 149.

9. Ogorodnikova, O. V., Mayer, M., Roth, J., J. Nucl. Mater., Vol. 313-316 (2003) 469.

10. A. Golubeva, V. Kurnaev, M. Mayer, J. Roth, in "HYDROGEN IN MATTER: A Collection from the Papers Presented at the Second International Symposium on Hydrogen in Matter (ISOHIM)", AIP Conference Proceedings Vol. 837 (2006), pp. 12-21

11. H. Iwakiri, K. Morishita, N. Yoshida, J. Nucl. Mater. 307-311 (2002) 135-138

12. García-Rosales, C., Franzen, P., Plank, H., Roth, J. and Gauthier, E., J. Nucl. Mater., 233-237 (1996) 803.

13. M. Poon et al., J. Nucl. Mater., 307-311 (2002) 723-728 
14. Van Veen, A., et al., J. Nucl. Mater., 155-157 (1988) 1113.

15. Poon, M., Deuterium trapping in tungsten, thesis, 2004. 